\title{
A Miniature Sensor for Measuring Reflectance, Relative Humidity, and Temperature: A Greenhouse Example
}

\author{
Reginald S. Fletcher, Daniel K. Fisher \\ Crop Production Systems Research Unit, Agricultural Research Service, United States Department of Agriculture, Stoneville, USA \\ Email: reginald.fletcher@ars.usda.gov
}

How to cite this paper: Fletcher, R.S. and Fisher, D.K. (2018) A Miniature Sensor for Measuring Reflectance, Relative Humidity, and Temperature: A Greenhouse Example. Agricultural Sciences, 9, 1516-1527.

https://doi.org/10.4236/as.2018.911106

Received: September 22, 2018

Accepted: November 27, 2018

Published: November 30, 2018

Copyright $\odot 2018$ by authors and Scientific Research Publishing Inc. This work is licensed under the Creative Commons Attribution International License (CC BY 4.0).

http://creativecommons.org/licenses/by/4.0/

\begin{abstract}
There is a growing interest in using miniature multi-sensor technology to monitor plant, soil, and environmental conditions in greenhouses and in field settings. The objectives of this study were to build a small multi-channel sensing system with ability to measure visible and near infrared light reflectance, relative humidity, and temperature, to test the light reflectance sensors for measuring spectral characteristics of plant leaves and soilless media, and to compare results of the relative humidity and temperature sensors to identical measurement obtained from a greenhouse sensor. The sensing system was built with off-the-shelf miniature multispectral spectrometers and relative humidity and temperature sensors. The spectrometers were sensitive to visible, red-edge, and near infrared light. The system was placed in a greenhouse setting and used to obtain relative reflectance measurements of plant leaves and soilless media and to record temperature and relative humidity conditions in the greenhouse. The spectrometer data obtained from plant leaf and soilless media were compatible with baseline spectral data collected with a hyperspectral spectroradiometer. The greenhouse was equipped with a relative humidity and temperature sensor. The relative humidity and temperature sensor measurements from our sensor system were strongly correlated with the relative humidity and temperature results obtained with the greenhouse sensors (i.e., correlation coefficients $>0.70$ or $<-0.70$ ), and the mean relative humidity and temperature sensor values were similar for our system and the greenhouse system. Overall, the proposed sensor showed good potential as a tool to measure spectral response patterns of plant and potting mix material and environmental conditions relevant to greenhouse research. The system was inexpensive to build; the total cost of its components was $\$ 123$.
\end{abstract}

\section{Keywords}

Arduino, Kale Red Russian, Relative Humidity, Soilless Media, Spectrometer, Temperature 


\section{Introduction}

Currently, miniature sensors have become common place in our everyday lives including triggering airbags in cars during a collision, monitoring excessive carbon monoxide concentration in homes and activating alarms when the values reaches a dangerous level, and monitoring weather conditions at weather stations [1]. They afford the user an opportunity to measure more than one variable, often required to meet industry and research initiatives.

Over time, the popularity of miniature multi-sensors has grown as tools for collecting agriculture data in greenhouses and in field settings. [2] monitored $\mathrm{pH}$, electrical conductivity, and temperature of tomato plant beds in a greenhouse with a small multi-sensor system. The Arduino microcontroller has become a staple for controlling a multitude of agricultural sensors because it is compact, inexpensive, and easy to use. [3] used it along with sensors to monitor soil-moisture status of field crops for irrigation scheduling and crop-water use studies, to measure daily evaporation-pan water levels for quantifying evaporative demand, and to monitor environmental parameters under forested conditions.

Rapid advances in technology have resulted in the development of inexpensive small-scale spectrometers capable of measuring visible, red-edge, and near infrared light. These regions of the spectrum are valuable for measuring plant health status and soil properties. There is a growing interest in using miniature multi-sensor technology to monitor plant, soil, and environmental conditions in greenhouses and in field settings, with an emphasis on collecting spectral reflectance data. It is hypothesized that a miniature multi-sensor system developed from inexpensive commercial products can adequately measure spectral reflectance properties of plant and soil material and relative humidity and temperature in a greenhouse setting. The objectives of this study were to build a multi-channel sensing system with the ability to measure visible and near infrared light reflectance, relative humidity, and temperature, to test the light reflectance sensors for measuring spectral characteristics of plant leaves and soilless media, and to compare results of the relative humidity and the temperature sensors to identical measurement obtained from a greenhouse sensor.

\section{Materials and Methods}

\subsection{Multi-Sensor Development}

A multi-sensor system was developed from off-the-shelf products (Figure 1). It contained the following components: 1) six-channel visible spectrometer (AS7262), 2) six-channel near infrared spectrometer (AS7263), 3) relative humidity sensor, 4) temperature sensor, 5) real-time clock, 6) microcontroller, 7) micro SD card holder, 8) multiplexer, and 9) rechargeable lithium ion battery. The six-channel visible and near infrared spectrometers are $4.5 \times 4.4 \mathrm{~mm}$ in size and are classified as ultra-low power consumption sensors. They have a 16-bit 


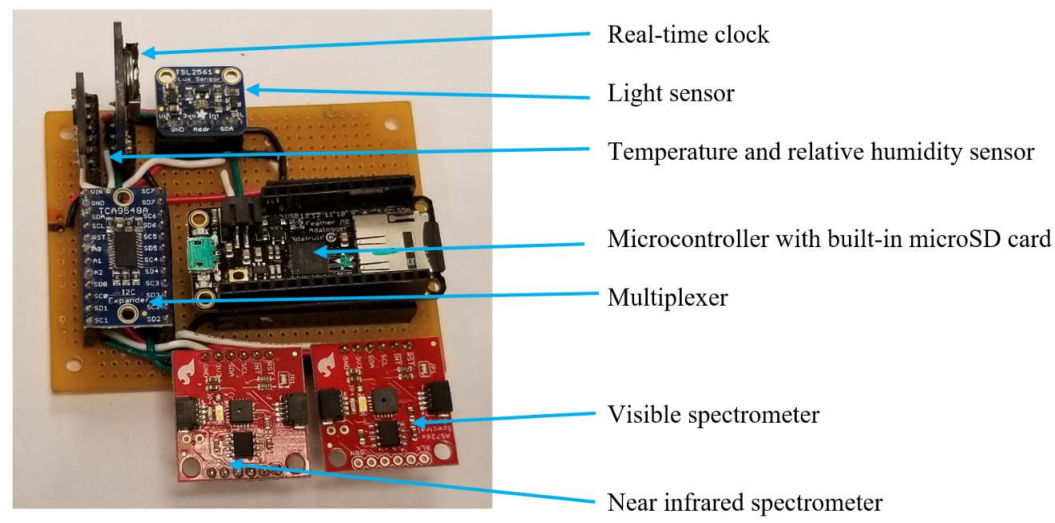

Figure 1. Sensing system components.

radiometric resolution. The sensors enable chip-scale spectral analysis by integrating Gaussian filters into standard complementary metal-oxide-semiconductor (CMOS) silicon via nano-optic deposited interference filter technology [4] [5]. The six-channel visible sensor is sensitive to the $400-700 \mathrm{~nm}$ spectral range with center wavelengths of $450 \mathrm{~nm}, 500 \mathrm{~nm}, 550 \mathrm{~nm}, 570 \mathrm{~nm}, 600 \mathrm{~nm}$, and 650 $\mathrm{nm}$. The full-width half-maximum of the sensors is $40 \mathrm{~nm}$. The six-channel near infrared sensor is sensitive to the $600-900 \mathrm{~nm}$ spectral range. Center wavelengths of the six sensors are $610 \mathrm{~nm}, 680 \mathrm{~nm}, 730 \mathrm{~nm}, 760 \mathrm{~nm}, 810 \mathrm{~nm}$, and 860 $\mathrm{nm}$. The full-width half-maximum of these sensors is $20 \mathrm{~nm}$. The visible and near infrared sensitive spectrometers are available in the form of breakout boards, which include sensors and auxiliary electronic components, from Sparkfun Electronics Inc. (Niwot, CO, USA). The AS7262 and AS7263 sensors communicate using the I2C two-wire protocol. Note that, in the field of remote sensing, $610 \mathrm{~nm}$ and $680 \mathrm{~nm}$ represent the visible red and red-edge region of the optical spectrum [6] [7].

The relative humidity and temperature sensors are grouped together in a compact metal-lid LGA package (BME280, Bosch Sensortec, Germany). The size and height of the sensor module is $2.5 \times 2.5 \mathrm{~mm}$ and $0.93 \mathrm{~mm}$, respectively. It is classified as a low power consumption device.

The real-time clock calendar breakout board (PCF8523, Adafruit Industries, New York, NY, USA) keeps track of time even if the microcontroller is reprogrammed or losses power. It supports communication using the I2C protocol and connection of multiple devices to the microcontroller via the same two input/output pins and two-wire interface.

The Feather M0 Adalogger (Adafruit Industries) development board consists of a programmable microcontroller and features a built-in microSD card holder. The Atmel ATSAMD21G18 ARM Cortex M0 is a 32-bit processor that operates at $48 \mathrm{MHz}$ and a $3.3 \mathrm{~V}$ power supply. The microSD card holder allows data storage to standard microSD cards, with a maximum memory size of $32 \mathrm{~Gb}$.

The Multiplexer (TCA9548A, Texas Instruments, Dallas, TX, USA) is a combination circuit that selects binary information from one of many input lines 
and directs it to an output line. The AS7262 and AS7263 spectral sensors both have identical I2C addresses and so cannot both be connected simultaneously to the same I2C two-wire interface. The multiplexer serves as a switching device, allowing the microcontroller to first communicate with one spectral sensor, then switch to communicate with the second sensor.

The digital light sensor (TSL2561, Adafruit Industries) provides precise calculations of lux and can be set up for different gain/timing ranges to detect light ranges from up to $0.1-40,000+$ Lux on the fly. It has both infrared and full spectrum diodes, meaning the user can separately measure infrared, full-spectrum, or human-visible light.

The sensing system is powered by a $3.7 \mathrm{~V}, 2500 \mathrm{mAh}$ rechargeable lithium ion battery (Adafruit Industries). At each measurement interval, sensor measurements and a date and timestamp are stored on the microSD card. A list of sensing system components and approximate costs is provided in Table 1 . The total cost of developing the multi-sensor system was approximately $\$ 123$. Electrical schematic, prototype board layout, and Arduino microcontroller program are all open-source and freely available from the authors upon request.

\subsection{Measurements with Plant and Potting Mix Sensors}

Two identical sensing systems were developed and tested for the study. The sensors were evaluated in a greenhouse located at the United States Department of Agriculture, Agricultural Research Service facility in Stoneville, MS, USA (Latitude: $33.425168^{\circ}$, Longitude: $-90.911875^{\circ}$ ). For clarity, the sensing systems are referred to as the plant sensor and the potting mix sensor.

The plant sensor was used to record the spectral response of kale red Russian (Brassica napus L.) plant leaves. The plant was grown in the greenhouse. Kale red Russian has broad leaves, allowing us to simultaneously test the visible and near infrared six-channel sensors on the same leaf. During testing, the plant

Table 1. Cost of sensor components.

\begin{tabular}{cc} 
Components & Cost (US\$) \\
\hline AS7262 six-channel integrated visible spectrometer & 25 \\
AS7262 six-channel integrated near infrared spectrometer & 25 \\
Light sensor & 6 \\
Microcontroller board & 20 \\
Rechargeable battery & 15 \\
BME280 - relative humidity and temperature & 20 \\
Real-time clock calendar & 5 \\
Multiplexer & 7 \\
Total & 123 \\
\hline
\end{tabular}


sensor was placed approximately $20 \mathrm{~cm}$ above the leaf of interest (recently matured leaf).

The potting mix sensor was used to record the spectral response of wet commercial potting mix (Pro-Mix BX General Purpose Potting Mix, Premier Tech Horticulture, Rivière-du-Loup, QC, Canada). The potting mix was placed in a black seed tray with holes in the bottom. The seed tray was placed in a larger container without holes. Water was added to the larger container and allowed to seep through the holes located on the bottom of the seed tray to keep the soil moist. It was added at 3-day intervals.

For the spectral measurements, the sensors were mounted on a ring stand and were positioned approximately $20 \mathrm{~cm}$ from the leaf of choice on the kale red Russian plant and from the surface of the potting mix. The sensors were attached to a flexible cable allowing the user to adjust the sensors distance and view of the target. The sensing systems were programmed to record data at 15-min intervals for 24 hours a day. The plant sensor study was initiated on July 10, 2018. The potting mix study was initiated on August 10, 2018.

\subsection{Spectral Reflectance Measurements}

To provide a standard baseline for evaluating the spectral response of the six-channel spectrometers, hyperspectral reflectance measurements of the kale red Russian plant leaf of interest and of the potting mix were acquired with a contact probe attached to a FieldSpec 3 spectroradiometer (Malvern Panalytical $\backslash$ Analytical Spectral Devices, Boulder, CO, USA). It recorded information in the $350-2500 \mathrm{~nm}$ spectral range. The contact probe had its own light source. During the measurement, it was in direct contact with the leaf and the potting mix surface. The instrument was calibrated prior to collecting reflectance readings of the selected plant leaf or the potting mix surface. For these measurements, the contact probe was moved to three different regions within the area of interest for the leaf and potting mix surface. Note that the goal was to collect the data in the region viewed by the plant and soil sensors.

Post-processing of the hyperspectral data included the following: 1) splice correcting the reflectance data, 2) averaging the three spectral measurements obtained from the plant or potting mix, 3) aggregating the data to $10-\mathrm{nm}$ bandwidths, and 4) removing spectral bands below $400 \mathrm{~nm}$ and greater than 1000 $\mathrm{nm}$. Splice correction and spectral averaging were completed with the ViewSpec Pro Software (Version 6.2, Malvern Panalytical\Analytical Spectral Devices). Data aggregation and removal of spectra were completed with $\mathrm{R}$ software $(\mathrm{R}$ version 3.5.1, Feather Spray [8]).

\subsection{Relative Humidity and Temperature Sensors}

The greenhouse (Micro Grow Sensor, Greenhouse Systems Inc., Temecula, CA) contained its own relative humidity and temperature sensors. Those sensors were used as a baseline to compare the relative humidity and temperature sen- 
sors measurements obtained by the plant and potting mix sensors. For this test, relative humidity and temperature sensor data were recorded manually from the greenhouse sensors since those sensors were not automated to collect continuous measurements. Measurements were recorded at 30-min intervals between the hours of 8:00 am to 4:00 pm.

\subsection{Statistical Analyses}

Pearson correlation [9] was used to assess the relationship between the data recorded by the plant, the potting mix, and the greenhouse relative humidity and temperature sensors. Correlations were considered statistically significant at $\mathrm{p} \leq$ 0.05 . Analysis of variance (ANOVA) was calculated to determine if differences existed among the means of relative humidity and temperature sensors. Tukey's test was tabulated to determine which means were statistically different. For ANOVA and Tukey tests, differences were considered statistically significant if $\mathrm{p}$ $\leq 0.05$. Analyses was completed with the R software.

\section{Results}

\subsection{Plant Spectral Reflectance Properties}

Figure 2 shows an image of the kale red Russian plant and the hyperspectral reflectance properties of one of its leaves. The spectral curve was representative of the typical spectral curve of a green plant leaf. In the visible region of the optical spectrum, the maximum reflectance occurred at $550 \mathrm{~nm}$, representing reflection of green light by plant chlorophyll, whereas, the lowest reflectance occurred at approximately $450 \mathrm{~nm}$ and $650 \mathrm{~nm}$, representing strong chlorophyll absorption in the blue and the red regions of the optical spectrum, respectively. At approximately $700 \mathrm{~nm}$, the reflectance increased sharply and then plateaued at $760 \mathrm{~nm}$, in the near infrared region of the spectrum. The region between 680 $\mathrm{nm}$ to $750 \mathrm{~nm}$ is referred to as the red-edge, which is commonly used to monitor vegetation stress.

The multispectral spectral regions observed by the six-channel integrated visible spectrometer are represented by the gray bars in Figure 2. Its sensors thoroughly cover the visible region of the spectrum. For a green leaf, the user might expect the greatest value to be recorded by the $550 \mathrm{~nm}$ sensor and the lowest values to be recorded in the strong chlorophyll absorption regions at $450 \mathrm{~nm}$ and $650 \mathrm{~nm}$. For six different test dates, those qualities were generally observed for the kale red Russian leaf measurements obtained between the hours of 11:00 am and 3:00 pm by the six-channel visible spectrometer of the plant sensor (Table 2).

The multispectral spectral regions observed by the six-channel integrated near infrared spectrometer are illustrated by the red bars in Figure 2. Its sensors records data in the visible, red-edge, and near infrared regions of the light spectrum. Based on the hyperspectral data, the user would expect the lowest values to occur at $610 \mathrm{~nm}$ and the highest values to be recorded at $760 \mathrm{~nm}, 810 \mathrm{~nm}$, or 860 $\mathrm{nm}$. For six separate dates, that general trend was observed for spectral 


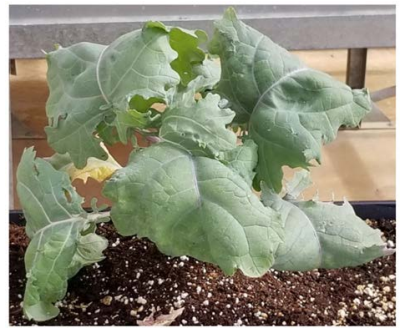

(a)

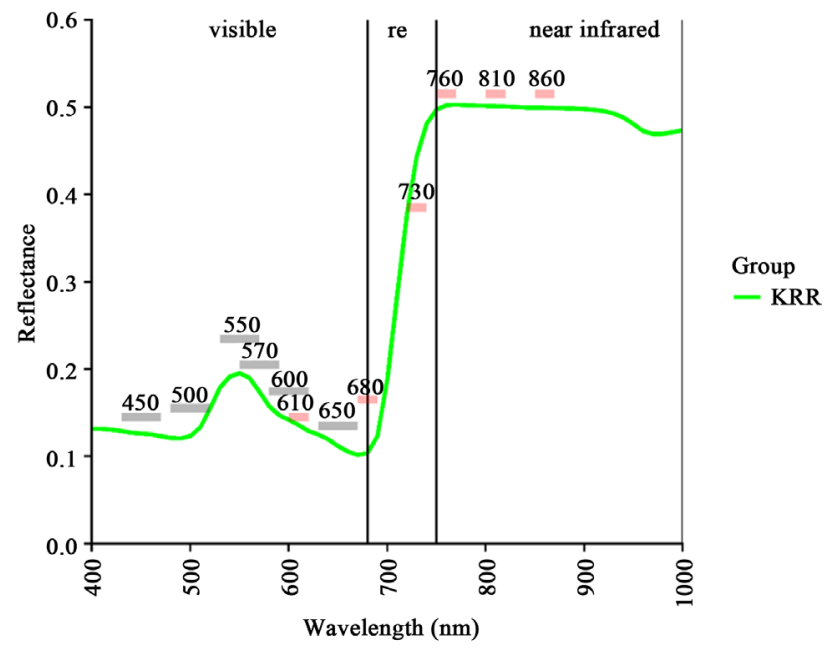

(b)

Figure 2. (a) Image of kale red Russian plant; (b) Hyperspectral reflectance (green line) curve of kale red Russian mature leaf (KRR) obtained with a contact probe attached to a spectroradiometer. Gray bars and numbers in the graph represent the $40-\mathrm{nm}$ wavelength and the center wavelength of the integrated six-channel visible spectrometer, respectively. Red rectangles and numbers show the 20 -nm wavelength and center wavelength, respectively, of the integrated six-channel multispectral near infrared spectrometer. re $=$ red edge.

Table 2. Average digital count values of kale red Russian leaf recorded by the integrated six-channel visible and near infrared spectrometers \pm 2 hours of solar noon.

\begin{tabular}{cccccccc}
\hline Spectrometer & Date & $450 \mathrm{~nm}^{\mathrm{a}}$ & $500 \mathrm{~nm}$ & $550 \mathrm{~nm}$ & $570 \mathrm{~nm}$ & $600 \mathrm{~nm}$ & $650 \mathrm{~nm}$ \\
\hline Visible & $7 / 10 / 2018$ & 12,408 & 14,986 & 18,750 & 17,325 & 9450 & 5093 \\
& $7 / 11 / 2018$ & 12,621 & 16,069 & 19,380 & 17,457 & 13,463 & 11,728 \\
& $7 / 12 / 2018$ & 9032 & 11,250 & 13,423 & 12,874 & 9,665 & 7370 \\
& $8 / 10 / 2018$ & 11,387 & 15,229 & 16,944 & 16,100 & 11,219 & 11,048 \\
& $8 / 13 / 2018$ & 5042 & 9693 & 10,543 & 9431 & 5710 & 5628 \\
& $8 / 16 / 2018$ & 9263 & 16,802 & 16,257 & 16,097 & 8612 & 11,014 \\
& & $610 \mathrm{~nm}$ & $680 \mathrm{~nm}$ & $730 \mathrm{~nm}$ & $760 \mathrm{~nm}$ & $810 \mathrm{~nm}$ & $860 \mathrm{~nm}$ \\
Near-infrared & $7 / 10 / 2018$ & 5093 & 5246 & 13,840 & 20,650 & 19,440 & 16,890 \\
& $7 / 11 / 2018$ & 5069 & 5098 & 12,641 & 18,581 & 18,657 & 17,042 \\
& $7 / 12 / 2018$ & 4688 & 4041 & 14,491 & 20,599 & 19,977 & 16,368 \\
& $8 / 10 / 2018$ & 5979 & 5698 & 10,004 & 15,295 & 11,453 & 9868 \\
& $8 / 13 / 2018$ & 4113 & 3839 & 4610 & 5706 & 7275 & 6651 \\
& $8 / 16 / 2018$ & 6292 & 6378 & 12,045 & 15,260 & 13,821 & 11,857 \\
\hline
\end{tabular}

${ }^{\mathrm{a} C e n t e r}$ wavelength for each sensor. 
measurements obtained with the six-channel near-infrared spectrometer of the selected kale red Russian leaf (Table 2).

\subsection{Potting Mix Spectral Reflectance Properties}

Illustrated in Figure 3 is the image of the potting mix and its hyperspectral reflectance curve obtained with a contact probe attached to the hyperspectral spectroradiometer. The potting mix's hyperspectral reflectance curve mimicked a hyperspectral reflectance curve of a typical soil. The lowest hyperspectral reflectance value was observed at $400 \mathrm{~nm}$ in the visible region of the optical spectrum, and the maximum hyperspectral value was detected in the near infrared region of the optical spectrum. For the six-channel visible and near infrared spectrometers, the user should expect to see a steady increase in the spectral values. For two test dates, that general increase was observed for measurements obtained by the visible and near infrared spectrometers (Table 3 ) of the potting mix.

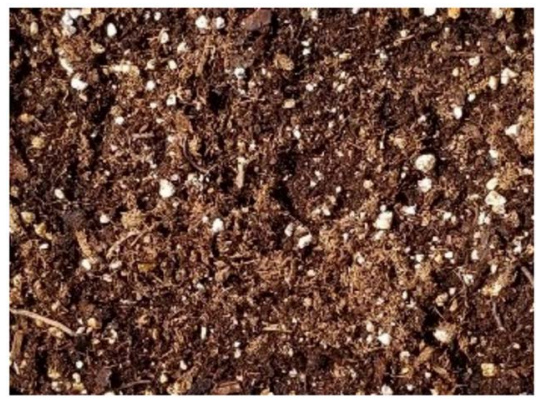

(a)

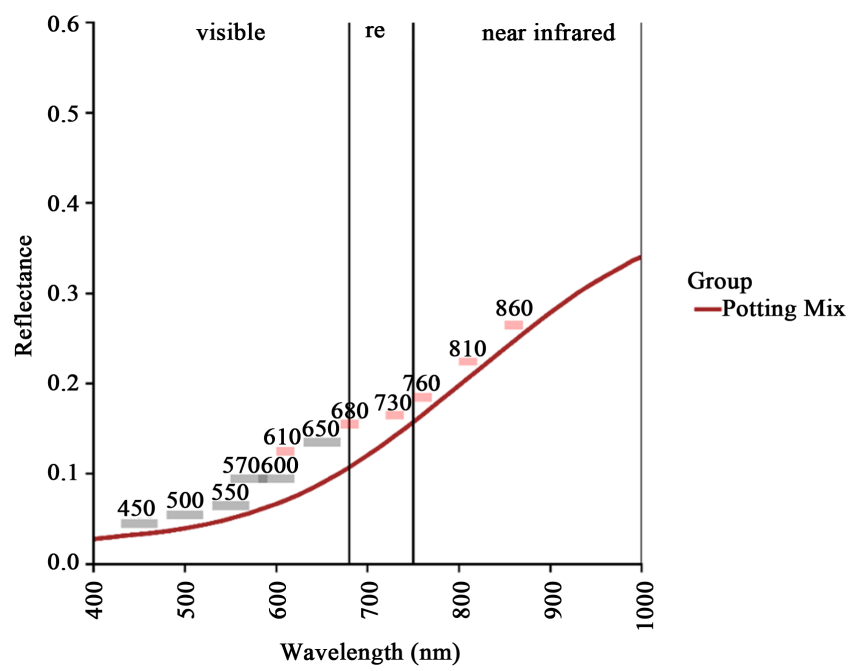

(b)

Figure 3. (a) Image of potting mix; (b) Hyperspectral reflectance (brown line) curve of potting mix obtained with a contact probe attached to a hyperspectral spectroradiometer. Gray bars and numbers on the graph represent 40 -nm wavelength and the center wavelength of the integrated six-channel visible spectrometer, respectively. Red bars and numbers show the $20-\mathrm{nm}$ wavelength and the center wavelengths, respectively, of the six-channel near infrared spectrometer. $r e=$ red-edge. 
Table 3. Average digital count values of potting mix recorded by the integrated 6-channel visible and near infrared spectrometers \pm 2 hours of solar noon.

\begin{tabular}{cccccccc}
\hline Spectrometer & Date & $450 \mathrm{~nm}^{\mathrm{a}}$ & $500 \mathrm{~nm}$ & $550 \mathrm{~nm}$ & $570 \mathrm{~nm}$ & $600 \mathrm{~nm}$ & $650 \mathrm{~nm}$ \\
\hline Visible & $8 / 10 / 2018$ & 2254 & 2673 & 2947 & 2935 & 3143 & 3617 \\
& $8 / 16 / 2018$ & 2303 & 2547 & 2892 & 2727 & 3385 & 3009 \\
& & $610 \mathrm{~nm}$ & $680 \mathrm{~nm}$ & $730 \mathrm{~nm}$ & $760 \mathrm{~nm}$ & $810 \mathrm{~nm}$ & $860 \mathrm{~nm}$ \\
Near-infrared & $8 / 10 / 2018$ & 1021 & 1454 & 2002 & 2208 & 2782 & 2580 \\
& $8 / 16 / 2018$ & 824 & 1114 & 1414 & 1800 & 2177 & 2092 \\
\hline
\end{tabular}

${ }^{a}$ Center wavelength for each sensor.

\subsection{Relative Humidity and Temperature Sensors}

For two different dates, Table 4 summarizes the correlation results of the temperature and relative humidity sensors. Statistically significant correlations $(\mathrm{p} \leq$ $0.05)$ were observed between the relative humidity and temperature sensor data. Overall, a negative relationship was observed between relative humidity and temperature. Using the greenhouse relative humidity and temperature sensors as the standard for comparison, high (correlation coefficients in the range of 0.70 to 0.89 or -0.70 to $-0.89,[10]$ ) to very high correlations (correlation coefficients in the range of 0.90 to 1.0 or -0.90 to -1.0 [10]) were observed between its sensors and the plant and the potting mix sensors relative humidity and temperature sensor readings.

To further substantiate the relative humidity and temperature sensor results, a one-way ANOVA was tabulated to determine if differences existed among measurements obtained with the plant, potting mix, and greenhouse sensors. Statistically significant differences were only observed among temperature sensor data recorded on August 16, 2018. For that date, the greenhouse temperature sensor value was statistically significant different than the plant and the potting mix temperature sensor values (Table 5).

\section{Discussion}

The six-channel visible and near infrared spectrometers' sensors were working properly. Their spectral response patterns were similar to the spectral response patterns observed for plant and potting mix reflectance measurements obtained with a spectroradiometer.

Reference [11], in the evaluation of hyperspectral narrowband data, identified twenty-five narrowbands (i.e., $\pm 5 \mathrm{~nm}$ ) that were not redundant and were useful for classification and modeling of vegetation or agricultural crops. Based on their results, a total of five channels for the six-channel visible and near infrared spectrometers combined would meet that criteria: $550 \mathrm{~nm}, 570 \mathrm{~nm}, 680 \mathrm{~nm}, 730$ $\mathrm{nm}$, and $850 \mathrm{~nm}$. Other wavebands meeting the criteria and falling within the sensitivity range of the six-channel visible spectrometer include $490 \mathrm{~nm}, 515 \mathrm{~nm}$, and $531 \mathrm{~nm}$. No additional bands listed by [11] were within the sensitivity range 
Table 4. Pearson correlation analyses of plant, potting mix, and greenhouse relative humidity and temperature sensors.

\begin{tabular}{|c|c|c|c|c|c|c|c|}
\hline Date & & $\mathrm{PRH}$ & $\mathrm{PT}$ & PMRH & PMT & GHRH & GHT \\
\hline \multirow[t]{7}{*}{$8 / 10 / 2018$} & PRH & & & & & & \\
\hline & PT & $-0.99^{*}$ & & & & & \\
\hline & PMRH & $0.86^{\star}$ & $-0.89^{*}$ & & & & \\
\hline & PMT & $-0.82^{*}$ & $0.87^{*}$ & $-0.99^{*}$ & & & \\
\hline & GHRH & $0.77^{\star}$ & $-0.79^{*}$ & $0.96^{*}$ & $-0.95^{\star}$ & & \\
\hline & GHT & $-0.75^{\star}$ & $0.81^{\star}$ & $-0.96^{*}$ & $0.98^{*}$ & $-0.96^{\star}$ & \\
\hline & & PRH & PT & PMRH & PMT & GHRH & GHT \\
\hline \multirow[t]{6}{*}{$8 / 16 / 2018$} & PRH & & & & & & \\
\hline & PT & $-0.97^{\star}$ & & & & & \\
\hline & PMRH & $0.93^{*}$ & $-0.86^{\star}$ & & & & \\
\hline & PMT & $-0.92^{*}$ & $0.85^{\star}$ & $-0.99^{*}$ & & & \\
\hline & GHRH & $0.91^{\star}$ & $-0.84^{\star}$ & $0.96^{*}$ & $-0.93^{\star}$ & & \\
\hline & GHT & $-0.80^{*}$ & $0.74^{\star}$ & $-0.81^{*}$ & $0.75^{*}$ & $-0.91^{*}$ & \\
\hline
\end{tabular}

PRH - Plant relative humidity sensor, PT_-Plant temperature sensor, PMRH-Potting mix relative humidity sensor, PMT-potting mix temperature sensor, GHRH-greenhouse relative humidity sensor, $\mathrm{GH}$ - greenhouse temperature sensor, ${ }^{*} \mathrm{p} \leq 0.05$.

Table 5. Mean comparison of plant, potting mix, and greenhouse relative humidity and temperature sensors.

\begin{tabular}{cccc}
\hline Date & Sensor & RH $(\%)$ & Temp (C) \\
\hline $8 / 10 / 2018(\mathrm{n}=10)$ & Plant & $59.4 \mathrm{a}$ & $32.9 \mathrm{a}$ \\
& Potting mix & $59.1 \mathrm{a}$ & $31.4 \mathrm{a}$ \\
& Greenhouse & $57.6 \mathrm{a}$ & $28.8 \mathrm{a}$ \\
$8 / 16 / 2018(\mathrm{n}=17)$ & Plant & $52.9 \mathrm{a}$ & $37.6 \mathrm{a}$ \\
& Potting mix & $52.6 \mathrm{a}$ & $36.4 \mathrm{a}$ \\
& Greenhouse & $51.4 \mathrm{a}$ & $31.9 \mathrm{~b}$ \\
\hline
\end{tabular}

RH-relative humidity, Temp-temperature. Values within a column for a particular date followed by different letters indicate a statistically significant difference between group at $\mathrm{p} \leq 0.05$ according to Tukey Honest Significant Difference Test.

of the near infrared spectrometer. According to the manufacturer bulletin [4] [5], the spectrometers can be fabricated to meet a user's specific need if so desired. In the future, potential users may want to have the manufacturer fabricate a visible spectrometer including the $490 \mathrm{~nm}, 515 \mathrm{~nm}$, or $531 \mathrm{~nm}$ spectral bands to enhance its use for vegetation study.

Overall, the potting mix spectra was a mixture of different materials. The potting mix consisted of sphagnum peat moss, perlite, vermiculite, limestone, and wetting agent. The sphagnum peat moss would be representative of the organic 
matter fraction of the potting mix. Perlite is an alumino-silicate ore of volcanic origin and is crushed to various particle sizes in the potting mix. Vermiculite is an expanded aluminum-iron, magnesium silicate mineral ore. Limestone is sedimentary rock consisting of calcium carbonate. The composition of the wetting agent was unknown. Comparable results were observed in the spectral response of the spectrometers and baseline spectrum response obtained with the hyperspectral spectroradiometer.

In a greenhouse setting, temperature and relative humidity are important components. They affect plant growth and productivity [12]. Increases in relative humidity are inversely correlated to decreases in temperature. High values of relatively humidity can lead to a reduction in plant growth. In contrast, low humidity values might cause an increase in plant dehydration. Generally, the temperature and the relative humidity information were similar between the fabricated multi-sensor systems and the greenhouse sensors, supporting the use of miniature relative humidity and temperature sensors in a greenhouse setting or other settings requiring automated, continuous temperature or relative humidity measurements.

Advantages and disadvantages were observed in the developed sensor platform. Using battery power, the six-channel spectrometers, relative humidity sensors, and temperature sensors collected data for at least seventy-two hours before changing the battery. We recommend checking the plant leaf for movement out of the sensor field of view at least twice a day, once in the morning and evening. Also, water the plant as needed because wilting of the plant leaf can cause it to move outside the sensors' field of view. For the relative humidity study, we noticed the plant and the potting mix sensors failed to collect data at specific times; missing data could be a glitch in the sensor or an error in the programming.

\section{Conclusion}

An integrated multimode sensing system for simultaneous measurements of spectral reflectance properties, temperature, and relative humidity was proposed. The system simultaneously obtained spectral reflectance, relative humidity, and temperature measurements and stored the data to a microSD card. The measurements recorded by the plant and potting mix multi-sensors were generally in agreement with baseline plant and potting mix spectra obtained with a spectroradiomter and relative humidity and temperature data obtained with a greenhouse sensor. Overall, the proposed sensing system showed good potential as a tool to measure spectral response patterns of plant and potting mix material and environmental conditions relevant to greenhouse studies. Future studies will focus on testing the spectral sensors on plants with different colored leaves, on evaluating the spectral sensors for measuring water quality, and on programming the light sensor to provide enough light for collecting data at night or in dark rooms. 


\section{Acknowledgements}

The authors would like to thank Milton Gaston, Jr., Keysha Hamilton, Destriale Jackson, Mekaela Brisco, and Zhaquavez Petty for their assistance in this study. Mention of a trade name, proprietary product, or specific equipment does not constitute a guarantee or warranty by the United States Department of Agriculture and does not imply approval of the product to the exclusion of others that may be available.

\section{Conflicts of Interest}

The authors declare no conflicts of interest regarding the publication of this paper.

\section{References}

[1] Brand, O. (2006) Microsensor Integration into Systems-on-Chip. Proceedings of the IEEE, 94, 1160-1176. https://doi.org/10.1109/JPROC.2006.873618

[2] Futagawa, M., Iwasaki, T., Murata, H., Ishida, M. and Sawada, K. (2012) A Miniature Integrated Multimodal Sensor for Measuring $\mathrm{pH}$, EC and Temperature for Precision Agriculture. Sensors, 12, 8338-8354. https://doi.org/10.3390/s120608338

[3] Fisher, D.K. and Gould, P.J. (2012) Open-Source Hardware Is a Low-Cost Alternative for Scientific Instrumentation and Research. Modern Instrumentation, 1, 8-20. https://doi.org/10.4236/mi.2012.12002

[4] AMS-TAOS USA Inc. (2016) Spectral ID a New Class of Spectral Sensing Products. AS7262-Integrated 6-Channel Visible Spectrometer Covering 400-700 nm Wavelengths. https://ams.com/documents/20143/36005/AS7262_FS000166_1-00.pdf

[5] AMS-TAOS USA Inc. (2016) Spectral ID a New Class of Spectral Sensing Products. AS7263-Integrated 6-channel Nir Spectrometer Covering 600-900 nm Wavelengths. https://ams.com/documents/20143/36005/AS7263_FS000163_1-00.pdf

[6] Horler, D.N.H., Dockray, M. and Barber, J. (1983) The Red Edge of Plant Leaf Reflectance. International Journal Remote Sensing, 4, 273-288. https://doi.org/10.1080/01431168308948546

[7] Jensen, J.R. (2000) Remote Sensing of the Environment: An Erath Resource Perspective. 2nd Edition. Prentice-Hall, New Jersey.

[8] R Core Team (2018) R: A Language and Environment for statistical Computing. R Foundation for Statistical Computing, Vienna. https://www.R-project.org/

[9] Asuero, A.G., Sayago, A. and Gonzalez, A.G. (2006) The Correlation Coefficient: An Overview. Critical Reviews in Analytical Chemistry, 36, 41-59. https://doi.org/10.1080/10408340500526766

[10] Zady, M.F. (2009) Correlation and Simple Least Squares Regression. https://www.westgard.com/lesson42.htm

[11] Thenkabail, P.S., Gumma, M.K., Teluguntla, P. and Mohammed, I.A. (2014) Hyperspectral Remote Sensing of Vegetation and Agricultural Crops. Photogrammetric Engineering and Remote Sensing, 80, 697-709.

[12] Prabhu, S.R., Gajendran, E. and Balakumar, N. (2016) Monitoring Atmospheric Conditions using Distributed Sensors. International Journal of Inventions in Engineering and Science Technology, 26, 108-120. 